\title{
Neutrophilic Dermatoses in a Seronegative Rheumatoid Arthritis Patient: a case report
}

\author{
Amel Awad IbnIdris Rodwan ${ }^{1}$, Abdel Gaffar. A. Mohammed ${ }^{2}$, Mohammed Elmujtba \\ Adam Essa ${ }^{3}$, Atif Elhadi Abdalla Babker ${ }^{4}$, Ali Mohamed Abdelsatir ${ }^{5}$, and Elnour \\ Mohammed Elagib ${ }^{6}$ \\ ${ }^{1}$ Sudan Medical specialization board \\ ${ }^{2}$ Security Forces Hospital Makkah \\ ${ }^{3} \mathrm{Al}$ Fashir University Faculty of Medicine \\ ${ }^{4}$ University of Gezira \\ ${ }^{5}$ Khartoum histocentre \\ ${ }^{6}$ Sudan Medical Specialization Board
}

November 23, 2021

\begin{abstract}
Rheumatoid arthritis (RA) is a chronic inflammatory disease, characterized by symmetric and destructive polyarthritis with a broad spectrum clinical manifestation of various organs. Rheumatoid neutrophilic dermatitis (RND) is an unusual distinctive manifestation of RA and typically develops in severe RA. 51-year-old female presented with a skin rash involving her extremities.
\end{abstract}

\section{Introduction}

RA is a common inflammatory synovial disorder characterized by symmetrical polyarthritis and associated with joint destruction $(1,2)$. RA has a broad spectrum clinical manifestation of various organs. Extraarticular findings and lesions due to skin involvement may also co-exist which include vasculitis, pyoderma gangrenosum, rheumatoid nodules and leg ulcers $(3,4)$. In addition to these findings, palmar erythema, urticaria, vitiligo, neutrophilic lobular panniculitis and RND may less commonly occur (5-7).

RND typically progresses in severe RA several years after the diagnosis of RA and manifests as symmetric erythematous and urticarial lesions over the extensor surfaces of the extremities (8). Dermatological involvement tends to occur in patients with more severe or active RA $(9,10)$. Here, we present a case of RND, a rare neutrophilic skin condition, in a seronegative RA Sudanese patient treated with cyclosporine.

\section{Case presentation}

A 51-year-old Sudanese female was diagnosed with RA in 2018 based on the EULAR classification of RA. She had a history of bilateral symmetrical polyarthritis with involvement of small joints of the hands, both wrists and shoulders; they are also associated with morning stiffness lasting for two hours. She was treated successfully with methotrexate and prednisolone and her disease remains in remission. A year ago, she started to develop an itchy skin rash with pus involving her lower extremities and abdomen. She had no fever, weight loss, skin rashes, bowel symptoms, aphthous ulcers or hair loss. Currently, she has no joint pain or extra-articular manifestations. Her systemic review was unremarkable. The patient had hypertension for 5 years, her family history revealed no history of connective tissue diseases. She is a mother of 7 healthy children with no history of abortion. In terms of drug history, she is on amlodipine $5 \mathrm{mg}$ for hypertension. Her musculoskeletal examination showed no synovitis, her skin examination showed nodulopapular lesions 
affecting the extensor surfaces of the lower extremities particularly on thighs and the lower part of the abdomen with hyperpigmentation as shown in the figures $(1,2)$. Laboratory tests revealed normal liver function test and renal function test, C-reactive protein $12.9(<5)$, Erythrocyte sedimentation rate (ESR) is $70 \mathrm{~mm} / \mathrm{hr}(<15)$, normal urine analysis and blood sugar level $98 \mathrm{mg} / \mathrm{dl}$. With regards to Immunological studies: Rheumatoid factor $(\mathrm{RF})<8$ (negative), anti-citrullinated peptide antibodies (ACPA) $<4(<20$, negative) and Antinuclear factor $<1 / 80$ (negative). Hand X-ray demonstrated periarticular osteopenia and the Chest x-ray was normal. Skin lesion biopsy showed multiple foci of necrosis, mixed inflammatory infiltrates composed mainly of neutrophils, hyperkeratosis and acanthosis and no evidence of vasculitis. The biopsy findings were consistent with a diagnosis of neutrophilic dermatosis fig $(3,4)$. The patient received topical and systemic steroids for the skin lesion however, no significant improvement was noticed, and then she was started on cyclosporin $100 \mathrm{mg}$ twice a day with close monitoring of the serum creatinine and blood pressure, few weeks later her skin lesions and general condition showed marked improvement.

\section{Discussion}

Dermatological complications in RA patients can be specific or related to skin disease due to the treatment taken by a patient (11). RA specific skin lesions are seen only in RA patients and include accelerated rheumatoid nodules, rheumatoid vasculitis, rheumatoid nodules and RND (8). Rheumatoid arthritis-related skin diseases include dermatoses that are associated with RA $(12,13)$. This group of skin disorders includes pyoderma gangrenosum, sweet's syndrome, Raynaud's phenomenon and erythema elevatum diutinum (EED). RND was first described by Ackerman in the last century. It is a rare and distinct manifestation of RA with high frequencies in females compared to males; only less than 20 cases had been described until now (14, 15).

The prevalence of RND is less than $2 \%$ among all RA patients (16). The exact pathophysiology of the condition is unknown; however, it is believed to be an immune-complex-mediated disease (17). RND commonly appears in patients with severe, chronic seropositive arthritis and clinically manifests as asymptomatic nodules, erythematous papules and urticaria-like lesions $(7,8)$. The dermatological lesion in RND can be either palpable purpura, or plaque or nodule in the Extensor surfaces of extremities and joints are favoured locations as in our presenting patient who showed nodulopapular lesions in the thighs and the lower part of the abdomen $(7,18)$. The differential diagnosis was considered and ruled out, which includes dermatitis herpetiformis ruled out by the absence of bullous lesions, A drug eruption was also ruled out because the lesions persisted and worsened in spite of cessation of medications, sweet syndrome was also excluded as the patient was not on any of the medications that can cause a Sweet's-like drug hypersensitivity (19). Behcet's disease, bowel-associated dermatosis arthritis syndrome and pyoderma gangrenosum were ruled Out by history and absence of ulcerative or vesiculopustular lesions. Erythema elevatum diutinum was ruled out by the absence of vasculitis (20).

Ethical approval and consent to publish

Obtained

\section{Conflict of Interest}

Non

\section{Written consent from the patient}

Obtained

\section{Funding}

No fund have been received

\section{Availability of data and materials}

All the data used in the study are available from the first and corresponding author on reasonable request 


\section{References}

1. Smolen JS, Aletaha D, McInnes IB. Rheumatoid arthritis. The Lancet. 2016;388(10055):2023-38.

2. Tilstra JS, Lienesch DW. Rheumatoid nodules. Dermatologic clinics. 2015;33(3):361-71.

3. Jagadeesan S, Shenoy P. Arthropathy in dermatology: A comprehensive review. Indian dermatology online journal. 2017;8(2):79.

4. Levin J, Werth VP. Skin disorders with arthritis. Best Practice \& Research Clinical Rheumatology. 2006;20(4):809-26.

5. Paget SA. Rheumatoid arthritis (RA) is a chronic systemic autoimmune and in-flammatory disorder of unclear etiology. It is characterized by a sym-metric polyarthritis, which is often erosive, resulting in irreversible damage to the joints. Although the musculoskeletal manifestations usually dominate the clinical picture, extra-articular manifestations are also common and on rare occasions can be life-threatening. $\mathrm{RA}$ is the most common immune-mediated inflammatory arthropathy, with a prevalence of up to $1.5 \%$ in North America. In some Native-American populations, the preva-lence may exceed 5\%. Expert Guide to Rheumatology. 2005:29.

6. Akdeniz N, Çalka Ö, Bilgili SG, Bayram İ. A Case of Rheumatoid Neutrophilic Dermatosis. Journal of the Turkish Academy of Dermatology. 2008;2(4).

7. Lazarov A, Mor A, Cordoba M, Mekori YA. Rheumatoid neutrophilic dermatitis: an initial dermatological manifestation of seronegative rheumatoid arthritis. Journal of the European Academy of Dermatology and Venereology : JEADV. 2002 Jan;16(1):74-6. PubMed PMID: 11952297.

8. Chua-Aguilera CJ, Möller B, Yawalkar N. Skin manifestations of rheumatoid arthritis, juvenile idiopathic arthritis, and spondyloarthritides. Clinical reviews in allergy \& immunology. 2017;53(3):371-93.

9. Hata T, Kavanaugh A. Rheumatoid arthritis in dermatology. Clinics in dermatology. 2006;24(5):430-7.

10. Bevin AA, Steger J, Mannino S. Rheumatoid neutrophilic dermatitis. Cutis. 2006 Aug;78(2):133-6. PubMed PMID: 16983904.

11. Lee HH, Song IH, Friedrich M, Gauliard A, Detert J, Röwert J, et al. Cutaneous side-effects in patients with rheumatic diseases during application of tumour necrosis factor- $\alpha$ antagonists. British Journal of Dermatology. 2007;156(3):486-91.

12. Marcucci E, Bartoloni E, Alunno A, Leone M, Cafaro G, Luccioli F, et al. Extra-articular rheumatoid arthritis. Reumatismo. 2018;70(4):212-24.

13. MATSUSHITA M, YAMAJI K, TAMURA N. Extra-articular Manifestations of Rheumatoid Arthritis. Juntendo Medical Journal. 2020;66(1):21-6.

14. Zuk G, Jaworecka K, Samotij D, Ostanska E, Reich A. Rheumatoid neutrophilic dermatitis. Reumatologia. 2019;57(6):350-3. PubMed PMID: 32226170. Pubmed Central PMCID: 7091481.

15. Below J, Helbig D. [Rheumatoid neutrophilic dermatitis]. Der Hautarzt; Zeitschrift fur Dermatologie, Venerologie, und verwandte Gebiete. 2015 Apr;66(4):228-30. PubMed PMID: 25757837. Rheumatoide neutrophile Dermatitis.

16. Ergun T, Inanc N, Tuney D, Kotiloglu EK, Seckin D, Tetik C, et al. Skin manifestations of rheumatoid arthritis: a study of 215 Turkish patients. International journal of dermatology. 2008;47(9):894-902.

17. Alavi A, Sajic D, Cerci FB, Ghazarian D, Rosenbach M, Jorizzo J. Neutrophilic dermatoses: an update. American journal of clinical dermatology. 2014;15(5):413-23.

18. Çetin GY, Sayarlığlu H, Erhan Ç, Kahraman H, Cıralık H, Sayarlıŏlu M. A case of neutrophilic dermatosis who develop palpable purpura during the use of montelukast. European journal of rheumatology. 


\section{4;1(4):170.}

19. Kumari I, Dongre A, Mohanty S. Rheumatoid Neutrophilic Dermatosis: The Distinct Entity with Florid Presentation. Indian dermatology online journal. 2020 Jan-Feb;11(1):114-5. PubMed PMID: 32055526. Pubmed Central PMCID: 7001406.

20. Wijenayake BK. Neutrophilic Dermatoses. Atlas of Dermatoses in Pigmented Skin: Springer; 2021. p. $213-23$.

\section{Figure legends}

Figure 1 : Shows a large nodule on the knee

Figure 2: shows a small papules and large crusted nodule

Figure 3 : Skin biopsy shows multiple foci of necrosis, mixed inflammatory infiltrate composed mainly of neutrophils .No evidence of vasculitis.

Figure 4 : Skin biopsy shows hyperkeratosis and acanthosis.

Fig (1):

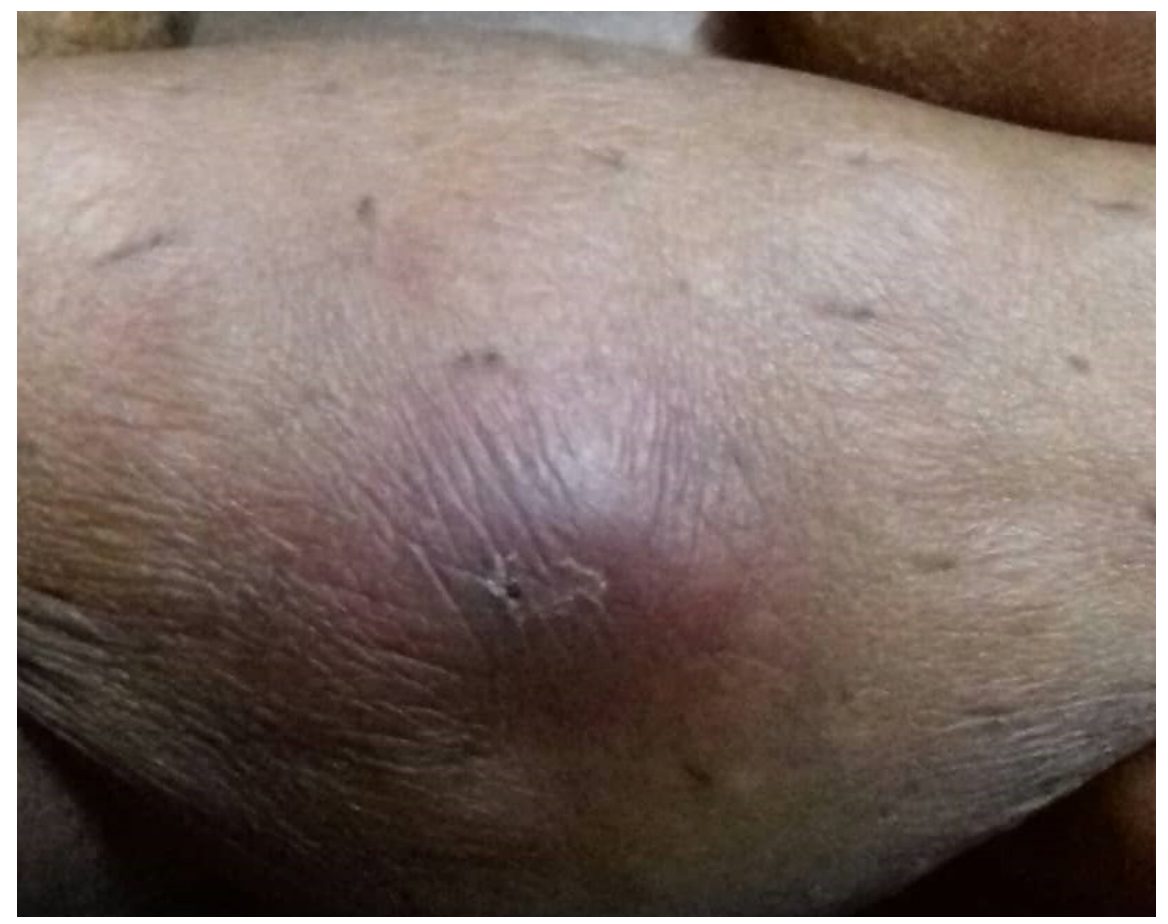

Fig (2): 



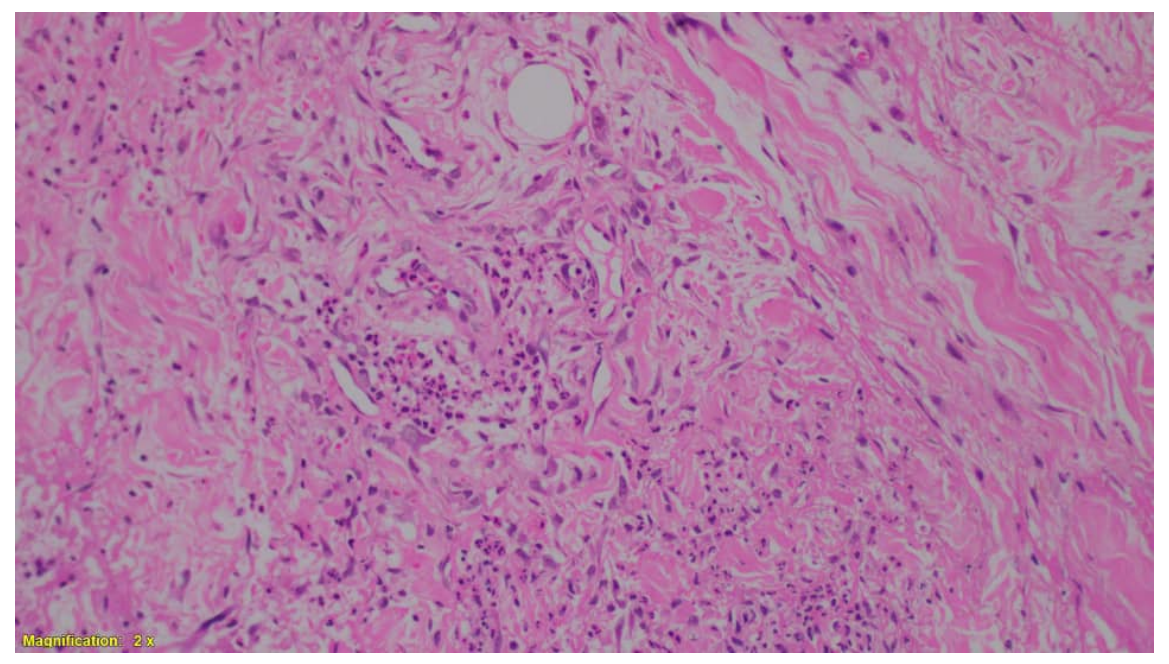

Fig (4):

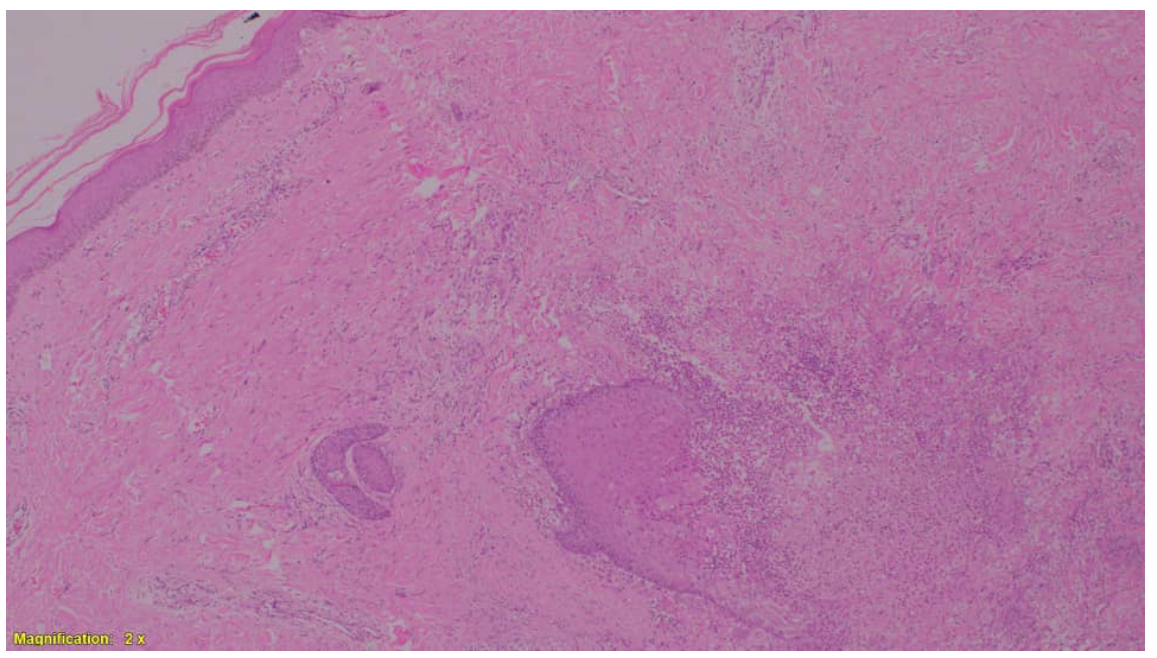

